\title{
Biodeterioro en rolletes de Pinus radiata preservados con sal CCA frente al ataque de hongos de pudrición parda
}

\author{
Bio-deterioration of Pinus radiata round wood preserved with CCA salt against brown rot fungi
}

\author{
Marco Torres $^{a *}$, Víctor Figueroa ${ }^{b}$, Isabel Vives ${ }^{c}$ \\ * A utor de correspondencia: a Universidad A ustral de Chile, Facultad de Ciencias F orestales y Recursos Naturales, \\ Instituto de Tecnología de Productos Forestales, Valdivia, Chile, tel.: 56-63-221224, mtorres2@uach.cl \\ b Universidad A ustral de Chile, Facultad de Ciencias Económicas y A dministrativas, Valdivia, Chile. \\ ' U niversidad A ustral de Chile, Facultad de Ciencias Forestales y Recursos N aturales, Valdivia, Chile.
}

\begin{abstract}
SUMMARY
In Chile, Pinus radiata wood has been classified as not durable according to Chilean standards NCh789. To increase its durability it is required to impregnate it with type CCA (copper, chromium, arsenic) soluble salts. The aim of this study was to analyse the effectiveness of the impregnation of five $P$. radiata round wood treated with this salt. From each $260 \mathrm{~cm}$ long round wood a disc was removed from one end, which withdrew a central strip that provided the samples for determination of preservative retention by calcinations and bio-deterioration through accelerated laboratory tests using two brown rot fungi, Gloeophylum trabeum and Poria placenta. It was observed, in the five samples of round wood, that the penetration of the preservative was irregular and pond shaped; covering between 40 to $80 \%$ of the total area, with little consistency in the retention and focusing on the periphery of discs. The preservative salt retention reached 4.3 to $10.5 \mathrm{~kg} \mathrm{~m}^{-3}$ on discs periphery, while in the inner zone, close to the pith, the retentions were 0.3 to $3.1 \mathrm{~kg} \mathrm{~m}^{-3}$. The specimens from the periphery of the round wood subjected to attack by xylophages fungi show ed a mass loss of $1 \%$, classifying the impregnated CCA wood of $P$. radiata as highly resistant and in the inner zone, close to the pith, mass loss reached $26 \%$, moderately resistant.
\end{abstract}

Key words: Pinus radiata round wood, retention, xylophages fungi, durability indexes.

\section{RESUMEN}

En Chile la madera de Pinus radiata ha sido clasificada como no durable según la norma chilena NCh789. Para aumentar su durabilidad se puede impregnar con sales hidrosolubles tipo CCA (cobre, cromo, arsénico). El objetivo del estudio fue analizar la efectividad de la impregnación de rolletes de $P$. radiata tratados con esta sal ante el biodeterioro. De cinco rolletes de $260 \mathrm{~cm}$ de largo se extrajo un trozo a $50 \mathrm{~cm}$ desde un extremo, del cual se retiró un listón central que proporcionó las probetas. Se determinó la retención del preservante mediante calcinación y el biodeterioro a través de ensayos acelerados de laboratorio, utilizando dos hongos de pudrición parda, Gloeophylum trabeum y Poria placenta. La penetración del preservante fue irregular y en forma de lagunas, cubriendo entre 40 y $80 \%$ del área total, con poca uniformidad en la retención, concentrándose esta en la periferia de los rolletes. La retención de la sal preservante en la periferia alcanzó 4,3 a 10,5 $\mathrm{kg} \mathrm{m}^{-3}$, mientras que en la zona interna, cercana a la médula, fue de 0,3 a 3,1 kg $\mathrm{m}^{-3}$. Las probetas de la periferia de los rolletes sometidas al ataque de los hongos xilófagos presentaron una pérdida de masa de $1 \%$, clasificando la madera impregnada de P. radiata con CCA como altamente resistente; en la zona interna, cercana a la médula, alcanzó una pérdida de masa de $26 \%$, clasificándose como moderadamente resistente.

Palabras clave: rolletes de Pinus radiata, retención, hongos xilófagos, índices de durabilidad.

\section{INTRODUCCIÓN}

Pinus radiata D. Don es una de las especies forestales de alto aprovechamiento económico en Chile por su participación en el mercado de la construcción. Sin embargo, es especialmente sensible al deterioro, por ello ha sido clasificada como una madera no durable según $\mathrm{NCh}$ 789 (INN 1987). Debido a esto, y para evitar la proliferación de agentes de deterioro, se usan productos químicos que impiden la acción de ellos (Quappe y M uñoz 2001). En Chile, la impregnación de la madera se efectúa prin- cipalmente con la sal tipo cobre-cromo-arsénico (CCA), producto químico formado por cobre (fungicida), cromo (fijador) y arsénico (insecticida). Estos elementos están presentes como una mezcla de óxido de cobre $(\mathrm{CuO})$, óxido de cromo $\left(\mathrm{CrO}_{3}\right)$ y pentóxido de arsénico $\left(\mathrm{As}_{2} \mathrm{O}_{5}\right)$, otorgando a la madera una durabilidad superior a 25 años. El mercado chileno todavía utiliza esta sal, a pesar de sus restricciones a nivel internacional (INFOR 2010). La madera cilíndrica (rolletes) de $P$. radiata impregnada es usada normal mente para viñedos, cercos, postes para agricul tura, pollos y pilotes, adquiriendo un color verde producto de 
la oxidación frente a los compuestos químicos de la madera (Lignum 2000, INN 2009). El rollete es el remanente central de una troza producto del proceso de debobinado. Además, por ser una madera juvenil duraminizada (1 a 10 anillos para árboles entre 14 y 33 años), el contenido de compuestos accesorios polifenoles (antocianinas, antoxantinas, ácidos hidroxibenzolico, glucócidos y otros) protege sólo parcialmente a esta del biodeterioro por hongos 0 insectos (Díaz-Vaz 2003).

La madera de $P$. radiata en estado natural y frente al ataque de hongos xilófagos como Gloeophyllum trabeum (Pers.) M urril. y Poria placenta (F ries) Cooke, ha sido clasificada como una madera moderadamente resistente (Juacida y Villanueva 1996, Sagardía 2000, Santander 2007), por lo que el uso de preservante se hace necesario para protegerla del biodeterioro según su uso.

En las últimas décadas, la impregnación de la madera en Chile ha cobrado mayor importancia y, debido a ello, existen 180 plantas impregnadoras, produciendo 450.000 $\mathrm{m}^{3}$ año-1. Estas plantas se ubican mayoritariamente en las regiones de Valparaíso, $\mathrm{O}^{\prime} \mathrm{H}$ iggins y M etropolitana. Sólo 20 plantas cuentan con certificación de calidad entregada por organismos tales como la Universidad del Bío Bío, Universidad de Talca, Fundación Chile y Corthorn (INFOR 2010).

La impregnación se realiza mediante el proceso de céIula Ilena (proceso Bethell) que introduce el preservante al interior de las células de la madera para que éstas sean inmunes a la acción de hongos, proporcionando un período de servicio no menos a cuatro veces el de su durabilidad natural (Junta Acuerdo Cartagena 1988).

El objetivo del presente trabajo es analizar rolletes de $P$. radiata impregnados con sal tipo cobre-cromo-arsénico para verificar el cumplimiento de la norma chilena $\mathrm{NCh}$ 819 (INN 2009), relacionada con la retención del producto y a través de un método acelerado de laboratorio verificar la pérdida de masa frente a la acción de dos hongos de pudrición parda.

\section{MÉTODOS}

Obtención de las muestras. Para el estudio se adquirieron en el comercio formal de Valdivia cinco rolletes de P. radiata impregnados con sal cobre-cromo-arsénico, y preservados según el método Bethell (Vásquez y Peredo 2005).

Para los ensayos de retención y biodeterioro de cada rollete $(260 \mathrm{~cm}$ de largo y diámetros entre 11 y $13 \mathrm{~cm}) \mathrm{se}$ extrajo una rodela de $5 \mathrm{~cm}$ de espesor a $50 \mathrm{~cm}$ de un extremo de acuerdo a la norma chilena N C h631 (IN N 2003). De cada rodela se obtuvo un listón diametral de $2,5 \mathrm{~cm}$ de ancho. De este listón se obtuvieron las probetas de ensayo de 2,5 x 2,5 x $1 \mathrm{~cm}$ en corte tangencial (figura 1). De una muestra de $P$. radiata de madera juvenil, se prepararon 10 probetas para los testigos, las que, junto al resto de las probetas fueron sometidas a ensayos de biodeterioro con los hongos de pudrición parda G. trabeum y P. placenta. EI número de probetas muestra para retención y biodeterioro por rollete fue de 27 .

Retención de sal preservante tipo cobre-cromo-arsénico. La cuantificación de la retención se hizo reduciendo en un molino las probetas hasta partículas de tamaño inferior a $1 \mathrm{~mm}$, las que se depositaron en un crisol para determinar el contenido de cenizas según norma TAPPI 211 (TAPPI 2000). Esta norma consiste en transformar la madera en aserrín, retirando porciones para determinar el contenido de humedad y para la calcinación a $575{ }^{\circ} \mathrm{C}$ por cuatro horas. Al finalizar dicho tiempo se dejó enfriar hasta $100{ }^{\circ} \mathrm{C}$, se retiraron los crisoles traspasándolos a un desecador, para posteriormente pesar el crisol con las cenizas.

Para los cálculos de retención se aplicó la siguiente fórmula [1]:

$\mathrm{QCCA}=(\mathrm{Cmcp}-\mathrm{Cmn}) \cdot \mathrm{Dm} \cdot 100^{-2}$

Donde:

QCCA = cantidad de óxidos activos de cobre-cromo-arsénico en la madera $\left(\mathrm{kg} \mathrm{m}^{-3}\right)$,

$\mathrm{Cmcp}=$ cenizas en madera con preservante cobre-cromoarsénico (\%),

$\mathrm{Cmn}=$ cenizas en madera natural $(\%)$,

$\mathrm{Dm}=$ densidad básica madera $\left(\mathrm{kg} \mathrm{m}^{-3}\right)$.

Para la determinación de cenizas en madera natural e impregnada se aplicó la siguiente fórmula:

Cenizas $(\%)=((\mathrm{Pccc}-\mathrm{Pc}) / \mathrm{Pm}) \cdot 100$

Donde:

Cenizas = contenido de cenizas con base en madera anhidra, $\mathrm{Pccc}=$ peso crisol con cenizas,

$P C=$ peso crisol,

Pm $=$ peso madera anhidra (aserrín).

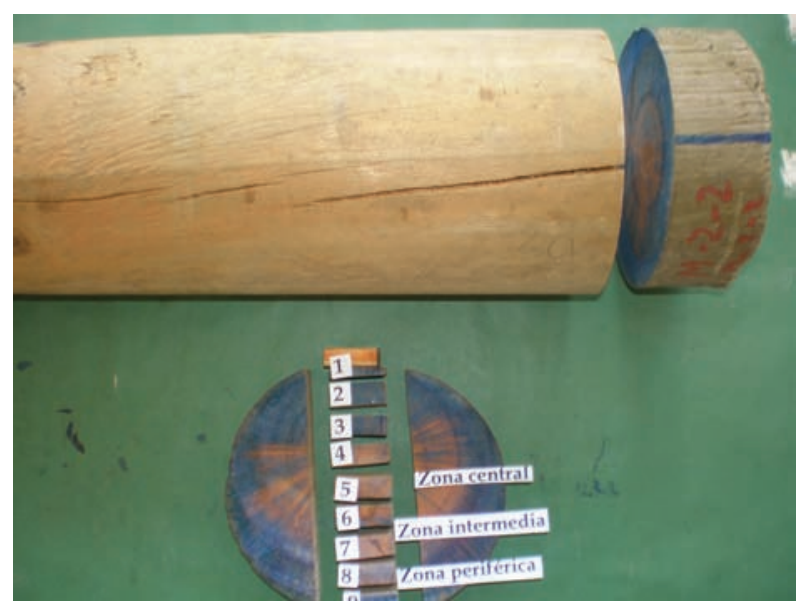

Figura 1. Extracción de muestras en el rollete. Sampling of round wood. 
Ensayo de durabilidad. La metodología utilizada se basó en la propuesta de la Oficina Federal para Pruebas de Materiales, BAM Berlín (Kerner-Gang 1984), que resume en forma corregida a DIN 52176 y ASTM D 1413-76 (Peredo 1988) basado en la pérdida de masa que experimenta la madera expuesta a la acción de hongos xilófagos. Se utilizó la clasificación propuesta por Findlay (1962) respecto a la resistencia natural de las maderas: altamente resistente $(0-10 \%$ de pérdida de masa), resistente (11 $24 \%$ ), moderadamente resistente $(25-44 \%)$ y no resistente $(>45 \%)$.

Para el ensayo de durabilidad se utilizaron las probetas descritas anteriormente. Cada probeta fue identificada y almacenada en una cámara de clima a $23 \pm 2{ }^{\circ} \mathrm{C}$ y $50 \%$ de humedad relativa, hasta que la madera alcanzó su humedad de equilibrio (18\%). Posteriormente se determinó la masa inicial de cada una de las probetas.

Hongos de prueba. Los hongos utilizados fueron G. trabeum y P. placenta suministrados por el Laboratorio de Patología Forestal de la Facultad de Ciencias Forestales y Recursos Naturales de la Universidad A ustral de Chile, a partir de cultivos puros desarrollados en agar extracto de malta al $2 \%$. Se decidió usar estos hongos debido a que están estandarizados internacionalmente (entre otros) para este tipo de ensayos y porque se observó en terreno que polines impregnados, cumpliendo la función de barreras camineras, presentaban frecuentemente pudrición parda.

Biodeterioro. Se prepararon 12 bandejas de aluminio de $200 \times 300 \times 40 \mathrm{~mm}$ que contenían el medio de cultivo formado por $93,7 \mathrm{~g}$ de vermiculita tamizada $2-3 \mathrm{~mm}$, $21,41 \mathrm{~g}$ de extracto de malta y $535,2 \mathrm{~mL}$ de una solución mezcla de ácido clorhídrico y cloruro de potasio $(50 \mathrm{~mL}$ de $\mathrm{HCl} 0,1 \mathrm{~N}$ y $950 \mathrm{~mL}$ de $\mathrm{KCl} 0,1 \mathrm{~N}$ ), para obtener un $\mathrm{pH}$ final del medio entre 3,5 y 4,0 (Wolff 1989). Una vez homogeneizada la mezcla de cultivo se instal aron sobre ésta listones de P. radiata de $8 \times 8 \times 100 \mathrm{~mm}$ como soporte de las probetas (Kerner-Gang 1984). En los extremos se colocaron listones de $P$. radiata de $10 \times 22 \times 50 \mathrm{~mm}$ como sustrato para el inicio del desarrollo del micelio de los hongos pudridores. $\mathrm{L}$ as bandejas fueron esterilizadas en autoclave a $121^{\circ} \mathrm{C}$ por 20 minutos. Posteriormente se inocularon con los hongos de prueba y se dejaron en incubación a $24{ }^{\circ} \mathrm{C}$ durante dos semanas, tiempo necesario para que los listones desarrollaran suficiente cantidad de micelio. Terminado este periodo se procedió a colocar sobre ellos las probetas de ensayo (10) y las probetas testigo (2) y se dejaron en estufa de cultivo a $24^{\circ} \mathrm{C}$ durante 16 semanas. Finalizado este período, se retiraron del medio de cultivo, se esterilizaron a $121{ }^{\circ} \mathrm{C}$ y se limpiaron cuidadosamente del micelio que las cubría. A continuación se llevaron a estufa de secado a $105^{\circ} \mathrm{C}$ para obtener el estado anhidro hasta peso constante y así realizar la segunda medición de pérdida de masa de las probetas.
Análisis estadístico. Se aplicó un análisis de varianza simple para la retención y pérdida de peso con cada hongo. Para la separación de medias se aplicó el método de las diferencias mínimas significativas (LSD de Fisher).

\section{RESULTADOS}

Retención de sal preservante tipo cobre-cromo-arsénico. Las retenciones promedio de óxido fluctuaron entre 2,17 y $3,51 \mathrm{~kg} \mathrm{~m}^{-3}$. La penetración del preservante en los rolletes fue periférica, irregular, en forma de lagunas sin uniformidad de este en las cinco rodelas. La retención máxima fue de $10,6 \mathrm{~kg} \mathrm{~m}^{-3}$ en la periferia de los rolletes, superando lo establecido por la Norma NCh 819 (INN 2009) que corresponde a $6,4 \mathrm{~kg} \mathrm{~m}^{-3}$, mientras que en su centro 0 zona de la médula la retención fue de $0,3 \mathrm{~kg} \mathrm{~m}^{-3}$. La distribución que alcanzó el preservante lo indicó el color azul al reaccionar el óxido de cobre con el indicador cromoazurol (figura 2).

La variación general de la retención fue de 1:7 en sentido radial. En la periferia se obtuvieron los valores más altos, tres a cuatro veces más que en el centro. Se observó una variación significativa radial con valores decrecientes desde la periferia hacia la médula. La sección central del rollete obtuvo val ores muy bajos de retención. Se observó una alta variabilidad (dispersión) en cada posición radial, particularmente en la periferia (figura 3).

El análisis de comparaciones de medias reflejó la conformación de cuatro grupos: periférico (zonas 1 y 9 del rollete), intermedio (zonas 2, 3, 4 y 7), central (médula o zonas 5 y 6), y un caso aislado correspondiente a la zona 8 (figura 3).

Pérdida de masa. La acción de pudrición de cada hongo fue mayor $(P<0,05)$ en las posiciones centrales del rollete; en cambio, hacia la periferia fue menor. La pérdida de masa por efecto de cada hongo varió significativamente

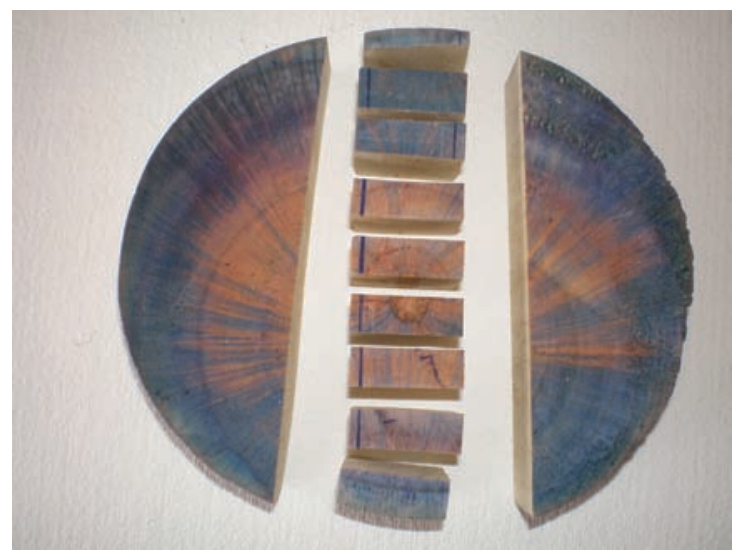

Figura 2. Penetración del preservante en los rolletes identificada por la coloración azul de la madera.

Preservative penetration in round wood identified by the blue colour of the wood. 


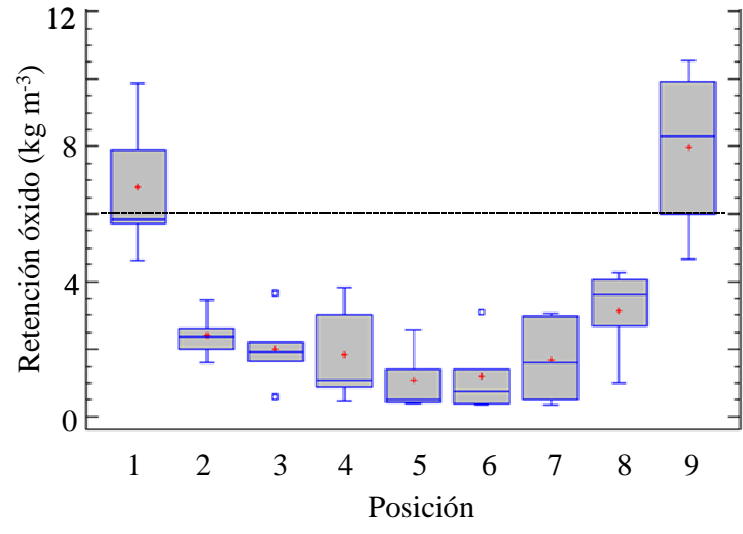

Exterior

Exterior

Figura 3. Retención de preservante según posición. Línea horizontal representa el mínimo exigido por la NCh 819.

Comparison of retention according to position. Horizontal line represents the minimum required by the NCh 819 .

entre las posiciones dentro del rollete. El análisis de comparaciones de medias, reflejó la conformación, para el caso de P. placenta, de tres grupos: de menor acción (periferia, zonas 1 y 9 , más las posiciones 2,3 y 4), de acción intermedia (zonas 5 y 8 ) y de máxima acción (parte central de rollete o médula, zonas 6 y 7) (figura 4A).

Con respecto a $G$. Trabeum, el comportamiento en la conformación de los grupos fue un tanto diferente, por cuanto se aprecian cinco agrupaciones, existiendo dos zonas, 1 y 2, de mínima acción del hongo. Por su parte, las posiciones $8,3,9,4$ y 5 conformaron un grupo homogéneo con una acción moderada y las posiciones 6 y 7, correspondientes a la parte central, del rollete donde se apreció la máxima acción del hongo (figura 4B).

En los testigos la pérdida de masa promedio fue similar para ambos hongos: $35,2 \% \pm 4,9 \%$ con G. trabeum y $35,7 \% \pm 4,3 \%$ con P. placenta.

\section{DISCUSIÓN}

A I impregnar la madera con sales tóxicas, y cumpliendo con la retención y penetración exigidas por la norma chilena, la madera ensayada por su heterogeneidad no clasifica como altamente resistente, obteniendo una durabilidad inferior a los 20 años. Los valores de retención de sal tipo cobre-cromo-arsénico, obtenidos en promedio en los rolletes, son menores a los recomendados por la Norma NCh 819 (INN 2009) que establece $6,4 \mathrm{~kg} \mathrm{~m}^{-3}$ de madera con un mínimo de $25 \mathrm{~mm}$ desde la superficie, según su uso.

L a heterogeneidad de la presencia de la sal en los rolletes estudiados indica que la zona de la médula no satisface la norma por lo que esta zona sería más susceptible de ser atacada por hongos cuando se agriete estando en uso, por lo tanto no sería recomendada para uso en exteriores y en contacto con el suelo pero sí puede ser usada como elemento estructural sobre el nivel el suelo.

Se observa que la retención, en general, en los rolletes logró valores cercanos al $50 \%$ de lo establecido por la norma. El efecto de superficialidad y escasa uniformidad de penetración del preservante se debe a las características físicas y químicas de la madera, ya que los rolletes son producto de la parte central de una troza que contiene madera juvenil duraminizada y un grado de permeabilidad baja (Kininmonth y Whitehouse 1991). Además la retención está influenciada por una adecuada aplicación del proceso Bethell (Vásquez y Peredo 2005). A través de los resultados obtenidos en la zona periférica, se deduce que la concentración del preservante es la adecuada pero su penetración es insuficiente y heterogénea. $\mathrm{Al}$ ser el rollete una madera juvenil duraminizada presenta oclusiones, baja permeabilidad y al to contenido de compuestos accesorios lo que es contraproducente para una buena impregnación subiendo los niveles de presión y tiempo. M ientras que al usar madera de raleo y zonas al tas del fuste que corresponde a madera juvenil, la exigencia en la impregnación es menor (Díaz-Vaz 2003).
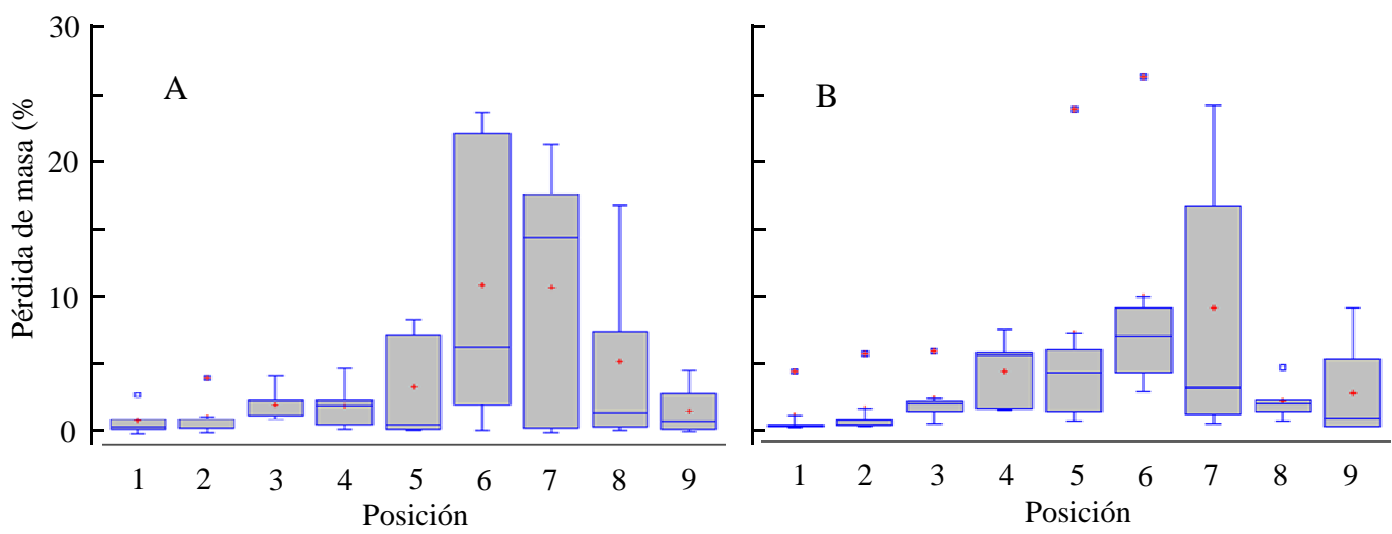

Figura 4. Pérdida de masa de la madera, según posición radial, por acción de A) P. placenta y B) G. trabeum. Comparison of weight loss according to position per share of A) P. placenta and B) G. trabeum. 
A menor retención de la sal tipo cobre-cromo-arsénico es mayor el porcentaje de pérdida de masa, coincidiendo en algunos casos con la pérdida de masa de los testigos sin preservante lo que clasificaría a esta madera como poco durable (Findlay 1962). La efectividad de ambos hongos pudridores, G. trabeum y P. placenta, es similar en pérdida de masa. L os hongos de pudrición parda utilizados en el ensayo actúan sobre los componentes químicos de la madera (holocel ulosa, lignina y extraíbles), nutriéndose preferentemente de azúcares y almidones que se encuentran en mayor concentración en la albura. Por el contrario, la madera de duramen contiene otro tipo de sustancias que son inhibidoras para el desarrollo de estos organismos como son los aceites esenciales, resinas, taninos, gomas, compuestos fenólicos, polifenoles y sustancias hidrosolubles diversas de alta toxicidad (Tuset y Durán 1979, Nicholas 1989).

Los valores obtenidos en el ensayo de biodeterioro se realizaron con madera de al bura por lo que al ser impregnadas con sal tipo cobre-cromo-arsénico le otorga un grado de toxicidad dejando la madera más resistente al ataque de hongos (Srinivasan et al. 1999, Goodell et al. 2007). Otros autores aseveran que esta inmunidad es debida a la presencia de componentes fenólicos en el duramen (K ininmonth y Whitehouse 1991), puesto que inhiben el desarrollo del micelio de los hongos pudridores.

Los hongos G. trabeum y P. placenta ensayados en este trabajo clasifican los rolletes sólo para uso en interiores (R1, R2 y R3) y no en exterior debido a que la exigencia de absorción de óxido para este uso es de 6,4 $\mathrm{kg} \mathrm{m}^{-3}$ (INN 2009).

La pérdida de masa de los testigos ratifica la validación del uso de los dos hongos siendo sus porcentajes de pérdida de masa similares a los obtenidos por Sagardía (2000) y Santander (2007), reafirmando al $P$. radiata sin preservante como madera no durable.

\section{CONCLUSIONES}

- La penetración y retención del preservante disminuye hacia el centro del rollete otorgando allí condiciones favorables al desarrollo de hongos, no así hacia la periferia que le otorga una al ta protección al biodeterioro.

- El efecto de superficialidad y escasa uniformidad de penetración del preservante se debe a las características químicas y morfológicas de la madera. También podría decirse que las condiciones de impregnación no fueron las óptimas.

- Los rolletes analizados no cumplen lo mínimo exigido por la norma chilena en su retención.

\section{REFERENCIAS}

Díaz-Vaz JE. 2003. Anatomía de maderas. Valdivia, Chile. Marisa Cuneo Ediciones. $151 \mathrm{p}$.

Findlay W. 1962. The preservation of timber. London, Inglaterra. A dam y Charles B lack. $162 \mathrm{p}$.

Goodell B, J Jellison, J Loferski, S Quarles. 2007. Brown-rot decay of ACQ and CA-B treated lumber. Forest Products Journal 57(6): 31-33.

INFOR (Instituto Forestal, CL). 2010. Tratamiento de preservación por doble vacío, una alternativa para la madera de pino utilizada en la construcción. Consultado 25 mar. 2010. Disponible en http://www.infor.cl/doble vacio/antecedentes. htm.

INN (Instituto Nacional de Normalización, CL). 1987. Norma Chilena Oficial 789/1.Of87. Maderas- Parte 1: Clasificación de maderas comerciales por su durabilidad natural. Santiago, Chile. IN N. 4 p.

INN (Instituto Nacional de Normalización, CL). 2009. Norma Chilena Oficial 819.Of09. Maderas preservadas - Pino radiata - Clasificación según uso y riesgo en servicio y muestreo. Santiago, Chile. INN. 19 p.

INN (Instituto de Normalización, CL). 2003. Norma Chilena Oficial 631.0f03. M adera preservada. Extracción de muestras. Santiago, Chile. INN. 7 p.

Juacida R, Villanueva J. 1996. Durabilidad natural de Sequoia sempervierens (D. D on) Endel. Bosque 17(1): 83-89.

Junta del Acuerdo Cartagena 1988. Manual del Grupo Andino para la Preservación de Maderas. PRID - Madera. Lima, Perú. Proyecto Subregional de Promoción Industrial de la Madera para Construcción. p. 12-29.

Kerner-Gang W. 1984. Prufüng des Schutzes von Holzspanplatten gegen Holzzerstörende Basidiomyceten im L aborverfahren. Holz-Zentralblatt 33: 509-511.

Kininmonth J, L Whitehouse. 1991. Properties and uses of New Zealand radiata pine. Volumen 1 wood properties. New Zealand. Forest Research I nstitute. $10 \mathrm{p}$.

Lignun 2000. Nuevas caras para el sector forestal. Fundación Chile. Santiago, Chile. Junio/julio 2000/46 - ISSN 0716 8624. p. 30-31.

Nicholas DD. 1989. Wood deterioration and its prevention by preservative treatments. N ew York, U SA. Syracuse U niversity Press. p. 89-92.

Peredo M. 1988. Fabricación de tableros de partículas para usos en exteriores. Bosque 9(1): 35-42.

Quappe J, M Muñoz. 2001. Impregnación de polines de roble (Nothofagus abliqua) y canelo (Drimys winteri). Consultado 10 may. 2010. Disponible en http://200.111.141.12:8081/ bn/archivos/archivos/Documentos/Impregnacion_polines_ roble_canelo.pdf.

Sagardía R. 2000. Estudio de impregnabilidad y durabilidad natural en madera de Populus $x$ Euroamericana (Dode) Guiniers. Tesis Ingeniero Forestal. Valdivia, Chile. Facultad de Ciencias Forestales, U niversidad A ustral de Chile. $72 \mathrm{p}$.

Santander K. 2007. Durabilidad natural de Acacia melanoxylon $R$. Brown. frente al ataque de hongos xilófagos. Trabajo de Titulación Ingeniero en M aderas. Valdivia, Chile. Facultad de Ciencias Forestales, U niversidad A ustral de Chile. $44 \mathrm{p}$.

Srinivasan U, T Ung, A Taylor, PA Cooper. 1999. Natural durability and waterborne preservative treatability of tamarack. Forest Products Journal 49(1): 82-87.

TA PPI (Technical A ssociation of Pulp and Paper Industry, USA ), 2000. A sh in Wood, Pulp, Paperboard: combustion at 525 ${ }^{\circ} \mathrm{C}$. Tappi T 211 om-93.

Tuset R, F Durán. 1979. Manual de maderas comerciales, equipos y procesos de utilización. M ontevideo, U ruguay. Hemisferio Sur. p. 505-506. 
Vásquez M, F Peredo. 2005. Impregnación de polines de híbridos de álamo tomando como referencia polines impregnados de pino. Bosque 26(3): 97-103.

Wolff J. 1989. Utilización del pino oregón (Pseudotsuga men- ziessi (M irb.) Franco) en la fabricación de tableros de partículas para el uso en exteriores. Tesis Ingeniero Forestal. Valdivia, Chile. Facultad de Ciencias Forestales, Universidad A ustral de Chile. 63 p.

Recibido: 10.05 .11

Aceptado: 24.08 .11 